\title{
Correction to: The cytidine deaminase under-representation reporter (CDUR) as a tool to study evolution of sequences under deaminase mutational pressure
}

Maxwell Shapiro ${ }^{1}$, Stephen Meier ${ }^{1}$ and Thomas MacCarthy ${ }^{1,2^{*}}$

\section{Correction}

Following publication of the original article [1], the authors reported that Figs. 1 and 3 were interchanged. The original article has been corrected.

The correct versions of the figures are given below:

Accepted: 21 June 2018

Published online: 04 July 2018

\section{Reference}

1. Shapiro M, et al. The cytidine deaminase under-representation reporter (CDUR)

as a tool to study evolution of sequences under deaminase mutational pressure. BMC Bioinformatics. 2018;19:163. 10.1186/s12859-018-2161-y

* Correspondence: thomas.maccarthy@stonybrook.edu

'Department of Applied Mathematics and Statistics, Stony Brook University, 100 Nicolls Road, Stony Brook, NY, USA

${ }^{2}$ Laufer Center for Physical and Quantitative Biology, Stony Brook University,

100 Nicolls Road, Stony Brook, NY, USA 


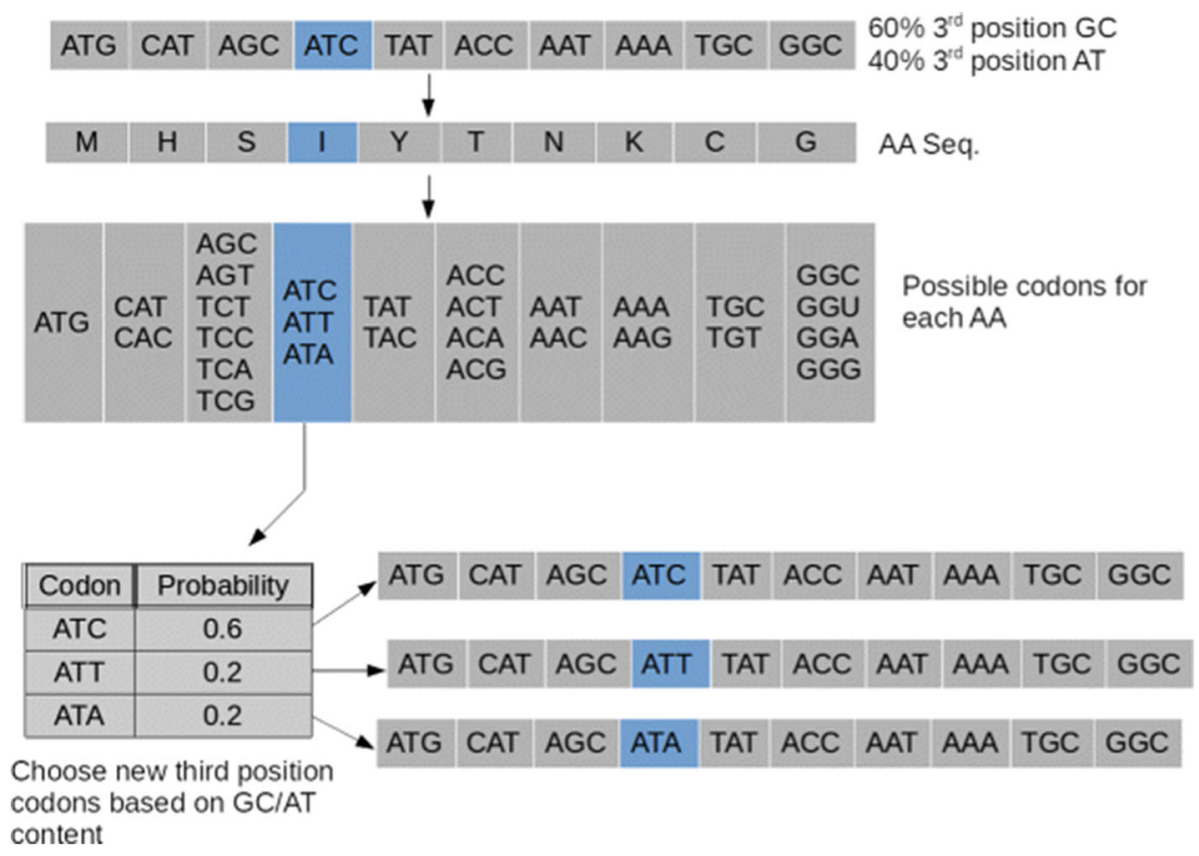

Fig. $1 \mathrm{gc} 3$ shuffle method. The choice of codons in the 4th nucleotide in the sequence (lle) was determined by the probabilities as follows: since there is an overall GC content of $60 \%$ at the 3rd position of the codons in the subject sequence, the ATC codon will be chosen with 0.6 probability. Since the AT content is then $\mathbf{0 . 4}$, the other two codons ATT and ATA are chosen randomly with equal probability, conditional on the $40 \%$ AT content. Note that the shuffling occurs iteratively throughout sequence, not just one codon at a time

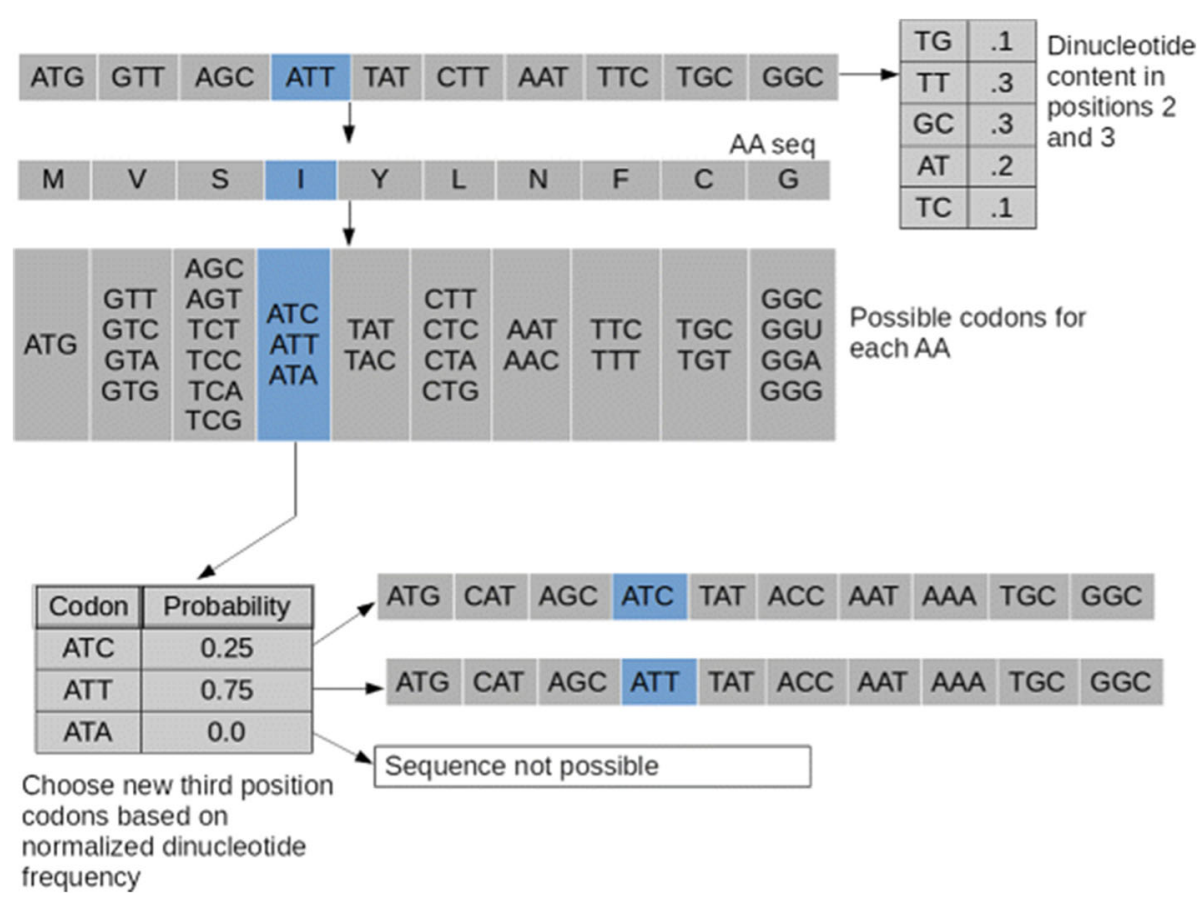

Fig. 3 dn23 shuffle method. First the dinucleotide frequency is calculated for the 2 nd and 3 rd codon positions of the original sequence. Then for each amino acid, codons are chosen based on the appropriately normalized probabilities for the dinucleotides available for that amino acid 